\title{
The World Wide Web's 25th anniversary
}

Twenty-five years ago, a proposal first laid out the principles of what was to become the World Wide Web.

In March 1989 at CERN, Tim Berners-Lee submitted his proposal to develop a radical new way of linking and sharing information over the internet. The document was entitled "Information Management: A Proposal" (CERN Courier May 2009 p24). And so the web was born. Now, Berners-Lee, the World Wide Web Consortium (W3C) and the World Wide Web Foundation are launching a series of initiatives to mark the 25th anniversary of the original proposal, and to raise awareness of themes linked to the web, such as freedom, accessibility and privacy.

Twenty-five years to the day after he submitted his proposal, on 12 March Berners-Lee, together with the Web Foundation, launched the "Web We Want" campaign. The aim is to promote a global dialogue and changes in public policy to ensure that the web remains an open, free and accessible medium, so that everyone around the world can participate in the free flow of knowledge, ideas and creativity online.

Berners-Lee announced the campaign at the Palais des Nations in Geneva on 10 December -Human Rights Day 2013 - during a series of conversations on a variety of issues in human rights, which were held in celebration of the 20th anniversary of the Office of the High Commissioner for Human Rights. There he set out the principles that inspire the movement for a free flow of information, such as affordable access, protection of privacy, freedom of expression, and neutral networks that do not discriminate against content or user.

Fittingly, the campaign is using the web to pass on the message, and it has already seen significant mobilization on social media with half a billion people worldwide hearing Berners-Lee's call for a digital bill of rights in every country. CERN promoted the launch of the campaign on its website with a series of opinion pieces from early contributors and enthusiasts of the World Wide Web, which are republished here.

For more aboutWeb@25 and the "Web We Want" campaign, visit www.webat25.org and webwewant.org.

\section{Résumé}

$25^{\text {ème }}$ anniversaire $d u$ World Wide Web

Vingt-cinq ans après avoir proposé le projet qui est devenu le World Wide Web, Tim Berners-Lee veut attirer l'attention sur certaines questions qui se posent dans ce domaine, telles que la liberté, l'accessibilité et la protection de la vie privée. Le CERN a soutenu le lancement de sa campagne sur son site web, en publiant les articles d'opinion reproduits ici.

Marina Giampietro, CERN.

\section{On the open internet and the free web}

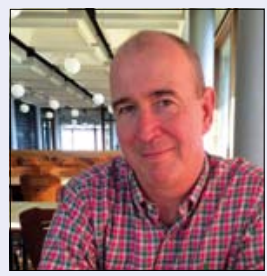

The internet created the platform and opportunity for people to communicate, to collaborate and to share at unprecedented scale and speed. The creation of the World Wide Web opened up these possibilities to the world, enabling individuals to participate and play their own creative role in the sharing of all human achievements.

This has enabled interactions between all sorts of people - from all sorts of domains, including business, government and scientific communities - for all manner of activities like never before in human history. The web has evolved from simple information sharing to transacting business through socializing and more recently collaborative problem solving in citizen cyber science. In these ways it harnesses the capabilities of humanity to do what we do best - share, learn, collaborate and innovate.

However, with this capability comes considerable responsibility. Basic human rights - including the right to freedom of expression and the protection of privacy - all need to be balanced and preserved in order that this incredible resource can be a safe and exciting place for creativity, for people of all ages and interests. The accessibility and openness of the internet are crucial to enabling new ideas to flourish and compete with long-standing traditions, and to ensure that the evolution of the web continues to proceed at a pace limited only by our ideas.

This responsibility rests with all of us - whether politicians, lawmakers, scientists or citizens - to ensure that the incredible progress we have made in the last 25 years, starting with the work of a few, and now capturing the innovations of many, can continue in an open, trusted, safe, free and fair way. - David Foster, Deputy Head of CERN's IT department. (Image credit: David Foster.) 


\section{Web@25}

\section{Minimizing the muddle}

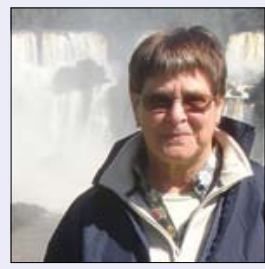

Reams of material have been written about where, why and when the World Wide Web was born, but what about its conception? Gestation was rather like that of an elephant - difficult to know it had started and taking almost two years to complete. In fact, I think the title of Tim Berners-Lee's book Weaving the Web, published in 1999 with Tim dubbed the inventor, is a better metaphor. When do a spider's first few threads become a web? And when, if ever, is the job finished?

In 1984, Tim was recruited by CERN's Data and Documents (DD) division and he elected to join the Read-Out Architecture (RA) section in the On-Line Computing $(\mathrm{OC})$ group. I was the RA section leader and Tim worked with (and without!) me for the next six years. Mike Sendall, the $0 \mathrm{C}$ group leader, agreed our work plans and held our purse strings.

At the time, CERN hosted lots of small and medium-sized experiments using a variety of mini-computers, personal computers, operating systems, programming languages and network links. Back at the ranch, the $\mathrm{OC}$ group was endeavouring to provide data-acquisition systems, the software used by equipment closely connected to the detectors, for as many experiments as possible. The conundrum, as in other areas, was how to embrace heterogeneity without having squads of workers generating exclusive solutions to intrinsically identical problems for bewildered users. Just the kind of anarchic jumble that Tim found challenging.

Several of us believed that standardization, where apt, reduced waste and frustration. But the s-word was anathema in some corners of CERN, on the grounds that it stifled creativity, and we evangelists incurred the wrath of a few mandarins. Yet conformity seemed to rankle less when it came to electronics. Commercial companies were already competitively producing computer interfacing hardware that conformed to ANSI/IEEE international standards.

Hurrah! If you know the hardware you're going to get, you can prescribe how to handle it. I had worked with the NIM (US)/ESONE (Europe) group that defined standard software routines for CAMAC interfacing and was on the committee developing hardware and software standards for the speedier
FASTBUS system. Tim arrived as we were dotting the Is and crossing the Ts of the FASTBUS routines.

He was obviously a smart young man (smart-clever rather than smart-sartorial!), full of fizz and, as a bonus, entirely likeable. When he presented his ideas in our section meetings, few of us if any could understand what he was talking about. His brain would overtake his voice, and holding up signs saying "Tim, slow down" rarely had the desired effect. We sometimes asked him to put things in writing, which didn't necessarily help either. One of his erstwhile colleagues recalls "we knew it was probably exciting, maybe even important, but that it could take hours to figure out". Listening to one of Tim's presentations today, one can still detect the run-away style, even after his training in public speaking. However, I remember an occasion when his delivery was impeccable, in a play performed by the Geneva English Drama Society!

Tim's main activity in the RA section was his Remote Procedure Call RPC, whereby a program on one computer could transparently access procedures, routines, on other computers, even if they used different operating systems and programming languages, and whatever the network connecting them. He wasn't too pleased when I asked him to specify the FORTRAN binding for FASTBUS routines, that is to define precisely the properties of the routines' parameters as seen from within a FORTRAN program. Only later did he appreciate the value of that unwelcome task, when preparing the standards that would underpin the first two Ws of WWW. He knew that the job, however tedious, had to be done and done well, with the devil lurking in the nit-picking details.

Come 1990, another CERN reshuffle and Tim stayed behind in the new Computing and Networks (CN) division, while the rest of us went off to Electronics and Computing for Physics (ECP). Shortly afterwards I drifted away from ECP, but I will always retain happy memories of the 1980 s and the pleasure of having Tim in our section. He was not the only singular character in that multifaceted team, but with his congenial personality he could work with anyone. At least I don't recall having to field any complaints, apart from "what on earth is Tim proposing?" Well, now we know.

- Peggie Rimmer, Tim Berners-Lee's supervisor from 1984 to 1990. (Image credit: Peggie Rimmer.)

\section{Good old Bitnet, and the rise of the World Wide Web}

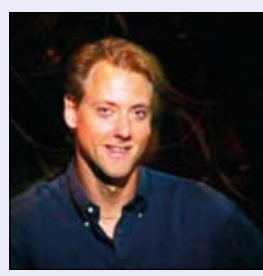

Although I presented my $\mathrm{PhD}$ thesis a mere 17 years ago, the last back-up of my thesis, programs and data was saved on a 7-inch magnetic tape reel. This of course meant that I did my graduate studies at the time when the word "network" was most often used in the plural. Each and every network was endowed with its own set of applications and accessibility for e-mail, document exchange, remote interactivity and even chatting.

Yes, computer-mediated social interaction came long before the World Wide Web. In the late 1980s, connectivity exploded at universities and research laboratories around the world. One noticeable side product was that young academics started dating each other from across the globe!
All of this was a heterogeneous mess, of course. But at the same time, it was pleasurably low level, and it was awesome. You knew what was happening behind the scenes when retrieving data and documents, you knew the hops that your "Relay" instant messaging made on the Bitnet, because you simply had to know. Data, documents, social interaction - it was all there. It was cool and in some ways efficient, but not practical, and it scaled very poorly.

And so the World Wide Web arrived on the internet. With the web came an immediate sense of need: you needed a fancy personal homepage, complete with graphical interface and colour. The personal homepage was quickly perceived as a way of asserting one's very existence. I was on a text-based, black-on-orange remote terminal, and I still remember putting together my first homepage late at night in early 1993 , while one of the 
graphical stations was free in the research group.

The web was practical and universal, and the other networks quickly withered away in a form of Darwinian selection. The web quickly drove the quest for desktop computer stations with screens with graphics capability. I still opted for size and sharpness, staying with black and white for several years, while all of my colleagues seemed to be rubbing their sandy eyes after only a few hours of 15 -inch colour experience.

The web brought a singular revolution that quickly changed every aspect of our screen work: a global, all-topic search possibility. Computer code, a formula, a result, a cooking recipe, a person, a phone number - everything was at hand in little more than an instant, with no physical displacement. We immediately started setting up analysis team pages to share progress more efficiently. I was in the DELPHI experiment Team 5, the "Higgs hunters" team. It was mostly pages with some expert documentation and links to plots, programs and data, but we also all invented countless ways to make information on the web dynamic. It took time and pain before it deserved the word interactive.
Today I sometimes have the impression that no development is ever made without constantly interrogating the web for advice, before even thinking through the problem: "Someone will surely have solved the problem in a better way, no?" is an all-too-common approach.

In those early days I rarely discussed my networked profession and life with friends and family - the web was just a new tool of my trade. After another long stay at CERN in 1993-1994, I went back to Stockholm in February 1994. Sitting quietly reading on the subway, it was with an indescribable surprise and awe for what was to come that I discovered an http address on a regular advertisement! Within months, commercial web addresses were all over our billboards in Sweden.

Back then, the good old Bitnet chat had a rule. The Dutch Master Operators insisted that "Relay is a 'privilege', NOT a right, and Relay abuse will NOT be tolerated!" I often wish the web had it too, including commercial boundaries under the same heading.

- Richard Jacobsson, senior physicist on the LHCb experiment. (Image credit: Alban Kakulya/STRATES.)

\section{Not at all vague and much more than exciting}

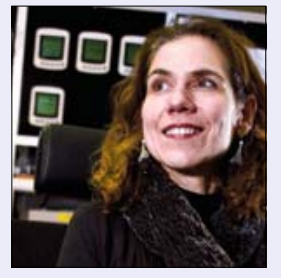

In 1989, when Tim Berners-Lee invented the World Wide Web at CERN, I was responsible for the laboratory's multi-protocol e-mail gateway. I remember discussing with Tim naming conventions for applications, and configuration rules for the first mailing lists that he requested to allow pioneer websites to discuss World Wide Web code.

We attended technical meetings sponsored by the European Commission - myself for e-mail standardization, and Tim for the Information Services Working Group (WG) - where he presented his code, and some Scandinavian universities even showed an interest in installing it.

Tim conceived, wrote and presented the web as an open, distributed, networked medium. He believed that the web should be accessible by everyone, everywhere - embracing from the first web conference at CERN in 1994 development for people with disabilities or a sub-optimal network infrastructure. He presented the web - in his proposal to CERN in March 1989 - as a platform for scientific collaboration, and 20 years later reinforced this commitment, announcing http://webscience.org as a home for scientists online.

And Tim Berners-Lee continues to strive for a free, open web today. Setting up the World Wide Web Foundation was just one of the many steps he took to maintain this ideal. On 12 May last year, at the United Nations in Geneva, Tim announced the Web We Want campaign, which will form the centre of the debate around today's information-surveillance methods.

As a CERN scientist, I share Tim's ideas for an open, collaborative web. I believe that CERN's software development based on web standards should be linked to the relevant working groups in the World Wide Web Consortium (W3C) - the main international standards organization for the World Wide Web.

Up until 1998, in the Web Office at CERN, we were still able to count all the world's web servers. We still thought we could keep track of the web's expansion. Apache put an end to this, as starting one's own web server became so easy. But we were still writing search algorithms of our own, with integrated dictionaries for natural language searches, with help from technical students. We enjoyed, at the time, a certain pluralism, because we had multiple commercial or public-domain products to compare and evaluate, search engines, web calendars and editing tools. We didn't use "Google" as a synonym for "search".

The explosion of websites around the turn of the century highlighted the importance of identifying trustworthy information online. At CERN, we understand that presence on the web doesn't necessarily make information valid - it must be recent and from a trusted source. Sophisticated algorithms are developed to promote web content by devious means, such as clever use of metadata to "arrange" the importance of search results, spread false rumours, manipulate public opinion. Browsing today requires a discerning eye and a knack for research.

Today, CERN software developers write grid middleware, data-management software, collaborative tools, repositories for data-preservation projects and web-based applications. They use, among other standards, the http protocol. A collaboration with relevant W3C working groups would lead to technical benefits in these times when resources are limited and the web has become much more than a document repository.

The web has changed human society more radically than Gutenberg's printing press. It is a valuable platform for education and free exchange of ideas. But it can also be a tool for propaganda and surveillance.

Now more than ever, we at CERN should keep in touch with the evolution of the web: after all, it changed the world as we know it at the end of the 1980s - it could do so again.

- Maria Dimou, CERN computer scientist and early web contributor. (Image credit: CERN-GE-0712019-01.) 


\section{GDOdFE//DU}

wwW.goodfellow.com

Metals

and materials

for research

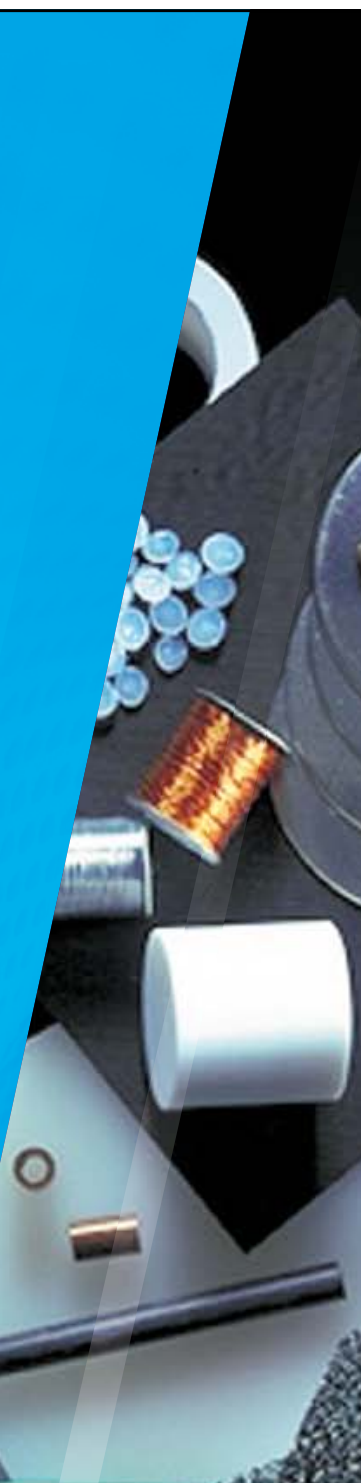

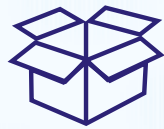

70000 PRODUCTS

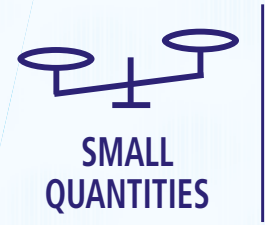

Goodfellow

Cambridge Limited

Ermine Business Park Huntingdon

PE29 6WR UK

Tel: 08007314653 or +441480424800

Fax: 08003287689 or +441480424900 info@goodfellow.com

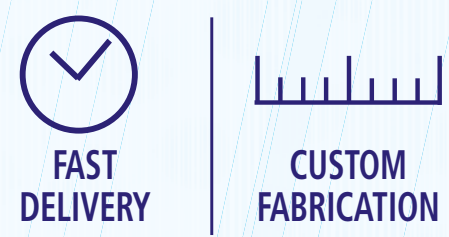

لسلسا CUSTOM
FABRICATION

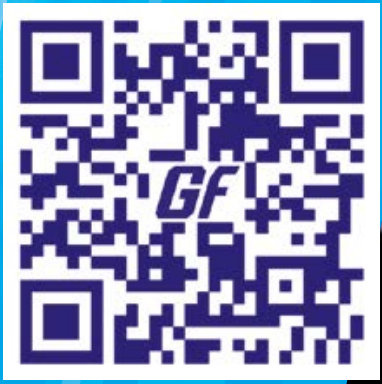

